\title{
3 Research Square

\section{Long non-coding RNA MALAT1 regulated ATAD2 to facilitate retinoblastoma progression by sponging miR-655-3p}

\author{
Yuxin Zhao \\ Weihai Central Hospital \\ Zhaoxia Wang \\ Weihai Central Hospital \\ Meili Gao \\ Weihai Central Hospital \\ Xuehong Wang \\ Weihai Central Hospital \\ Hui Feng \\ Weihai Central Hospital \\ Yuanyuan Cui \\ Weihai Central Hospital \\ Xia Tian ( $\square$ chengcai1997@126.com ) \\ Weihai Central Hospital
}

Research

Keywords: MALAT1, miR-655-3p, ATAD2, tumor progression, retinoblastoma

Posted Date: February 28th, 2020

DOl: https://doi.org/10.21203/rs.3.rs-15499/v1

License: (c) (i) This work is licensed under a Creative Commons Attribution 4.0 International License.

Read Full License 


\section{Abstract}

Background: Long non-coding RNA (IncRNA) metastasis associated lung adenocarcinoma transcript 1 (MALAT1) was reported as an oncogene in many tumors including retinoblastoma (RB). This research mainly focused on the functions and mechanism of MALAT1 in RB.

Methods: The levels of MALAT1, microRNA-655-3p (miR-655-3p), and ATPase family AAA domain containing 2 (ATAD2) in RB tissues and cells were measured by quantitative real-time polymerase chain reaction (qRT-PCR). The cell viability and apoptotic rate were monitored via cell counting kit 8 (CCK8) assay and flow cytometry, respectively. The protein levels of p21, CyclinD1, B-cell lymphoma-2 (Bcl-2), cleaved-casp-3, E-cadherin, Ncadherin, Vimentin, and ATAD2 were detected by Western blot assay. Transwell assay was performed to estimate the abilities of migration and invasion. The interactions between miR-655-3p and MALAT1 or ATAD2 were predicted by starBase. Dual-luciferase reporter assay was constructed to verify these interactions. The mice model experiments were established to validate the effects of MALAT1 in vivo.

Results: MALAT1and ATAD2 were significantly increased while the level of miR-655-3p was remarkably decreased in RB tissues and cells. MALAT1 knockdown inhibited cell proliferation, metastasis, and epithelial-mesenchymal transition (EMT) but promoted apoptosis via miR-655-3p in vitro, and blocked xenograft tumor growth in vivo. MALAT1 was validated to sponge miR-655-3p and ATAD2 was verified as a candidate of miR-655-3p. MiR-655-3p overexpression inhibited cell proliferation but promoted apoptosis by targeting ATAD2. MALAT1 silencing affected cell behaviors by regulating ATAD2. MALAT1 depletion down-regulated ATAD2 expression via miR-655-3p in RB cells.

Conclusion: MALAT1 positively regulated ATAD2 to accelerate cell proliferation but retard apoptosis by sponging miR-655-3p in RB cells.

\section{Background}

Retinoblastoma (RB), originated from the retina, is the most aggressive intraocular cancer in children and a big threat to the infant vision and lives [1]. With the improvement of treatment, the survival of RB patients is almost $100 \%$ [2]. To some extent, the high cure rate of RB is based on the accurate diagnosis in the early stage [3]. For example, the survival rate of RB in Africa was only $20-46 \%$ because of the diagnosis at advanced stage [4]. Thus, the searching for novel biomarkers is essential for RB patients.

Long non-coding RNAs (IncRNAs) are a category of long RNAs (> 200 nucleotides (nt)) with no translation capacity and could affect target gene expression at the transcriptional stage [5]. Many IncRNAs were reported to be dysregulated in RB and regulate biological processes in the tumor. For example, LINC00202 was highly expressed in RB, and its silencing retarded cell growth, metastasis in vitro [6]. THOR was upregulated in $\mathrm{RB}$, the depletion of THOR resulted in the decrease of cell growth, metastasis, and the increase of apoptosis [7]. Metastasis associated lung adenocarcinoma transcript 1 (MALAT1) is a highly conserved non-coding RNA. Amounting evidence indicated that the MALAT1 was highly expressed in 
various cancers, including ovarian cancer [8], gastric cancer [9], colorectal cancer [10], papillary thyroid cancer [11], and non-small cell lung cancer [12]. Whereas, the molecular mechanism of MALAT1 in RB was rarely reported.

MicroRNAs (miRNAs), a form of small RNAs ( 18-25 nt) without the ability of translation, were identified to influence the expression of the target gene by targeting message RNA (mRNA) [13]. The dysregulation of miRNAs was documented in RB. For instance, the low expression of miR-125a-5p in RB promoted cell growth [14]. The similar results about miR-3619-5p [6], miR-492 [15], and miR-758 [16] were also found in RB. MiR-655-3p has been reported to impact cell behaviors of tumor progression in ovarian cancer [17], non-small cell lung cancer [18]. ATPase family AAA domain containing 2 (ATAD2), located on human chromosome 8q24, is a member of the ATPase family containing an AAA domain which is vital for ATPase activity and protein assembly [19]. ATAD2 was reported to affect many biological processes in diverse types of tumors $[20,21]$. However, the mechanisms of miR-655-3p and ATAD2 were scarcely documented in RB. In this project, we aimed to elucidate the underlying mechanism of MALAT1 in RB progression and search novel biomarkers for RB patients.

\section{Materials And Methods}

\section{Tissues collection}

Thirty RB tissue samples were collected from Weihai Central Hospital, as well as eighteen normal globe tissue samples from children. All patients were not subjected to radiation therapy and chemotherapy. All tissues were frozen at $-80^{\circ} \mathrm{C}$ until further used. The research was permitted by the Ethics Committee of Weihai Central Hospital and carried out according to the Declaration of Helsinki Principles. Writing informed consents were provided by all patients.

\section{Quantitative real-time polymerase chain reaction (qRT-PCR)}

The random primers (TaKaRa, Dalian, China) were used to synthesize cDNA. An SYBR Mix (TaKaRa) was used to perform the quantitative PCR on a real-time detection system (Bio-Rad, Shanghai, China). The levels of MALAT1, ATAD2 were normalized by glyceraldehyde 3-phosphate dehydrogenase (GAPDH) while the level of miR-655-3p was normalized by small nuclear RNA U6, and processed by the $2^{-\Delta \Delta C t}$ method. The primers were obtained from Songon (Shanghai, China) and presented as follows: MALAT1, (F: 5'-AATGTTAAGAGAAGCCCAGGG-3', R: 5'-AAGGTCAAGAGAAGTGTCAGC-3'); miR-655-3p, (F: 5'CAATCCTTACTCCAGCCAC-3', R: 5'-GTGTCTTAAGGCTAGGCCTA-3'); ATAD2, (F: 5'-

GGAATCCCAAACCACTGGACA-3', R: 5'-GGTAGCGTCGTCGTAAAGCACA-3'); GAPDH, (F: 5'GGCATTGCTCTCAATGACAA-3', R: 5'-TGTGAGGGAGATGCTCAGTG-3') and U6, (F: 5'CTCGCTTCGGCAGCACA-3', R: 5'-AACGCTTCACGAATTTGCGT-3').

\section{Cell culture and transfection}


Two retinoblastoma cell lines Y79 and WERI-Rb-1 were purchased from SXBIO (Shanghai, China) and adult retinal pigment epithelial cell line ARPE19 was bought from Tongpai (Shanghai, China). All cells were cultivated in RPMI-1640 medium (Biosun, Shanghai, China) containing 10\% fetal bovine serum (FBS; Biosun) in $5 \% \mathrm{CO}_{2}$ incubator at $37^{\circ} \mathrm{C}$. Small interfering RNA (siRNA) targeting MALAT1 (si-MALAT1, 5'-CACAGGGAAAGCGAGTGGTTGGTAA-3') and its negative control (si-con, 5'-

UUCUCCGAACGUGUCACGUTT-3'), miR-655-3p mimics (miR-655-3p) and its negative control (miR-con), miR-655-3p inhibitor (anti-miR-655-3p) and its negative control (anti-miR-con), and ATAD2 overexpression vector (pcDNA-ATAD2) and empty vector ( $p c D N A)$ were all obtained from Sangon. The transfection was conducted using Lipofectamine 2000 (Invitrogen, Carlsbad, CA, USA).

\section{Cell counting kit 8 (CCK8) assay}

A CCK8 kit (Beyotime, Shanghai, China) was utilized to assess cell viability. The Y79 and WERI-Rb-1 cells $\left(2 \times 10^{4}\right.$ per well) were injected into a 96-well plate and maintained for $24 \mathrm{~h}$. After transfection, CCK8 solution was added into each well at $0 \mathrm{~h}, 24 \mathrm{~h}, 48 \mathrm{~h}$, and $72 \mathrm{~h}$. The cell viability was assessed at $450 \mathrm{~nm}$.

\section{Flow cytometry analysis of cell apoptosis}

An apoptosis analysis kit (Servicebio, Wuhan, China) was used to detect the apoptotic rate. In brief, the transfected Y79 and WERI-Rb-1 cells were firstly washed with phosphate buffer solution (PBS) for three times. Then cells were incubated with Annexin V fluorescein isothiocyanate (FITC) and propidium iodide (PI) for $15 \mathrm{~min}$ in dark condition. The apoptotic rate was assessed using flow cytometry (Agilent, Beijing, China).

\section{Western blot assay}

The protein in Y79 and WERI-Rb-1 cells was extracted using RIPA buffer (Solarbio, Beijing, China) and the concentration of protein samples was detected using BCA Protein Assay Kit (Beyotime). After being separated by sodium dodecyl sulfonate-polyacrylamide gel electrophoresis (SDS-PAGE), the protein samples were transferred onto a polyvinylidene fluoride (PVDF) membrane (Millipore, Billerica, MA, USA). The membrane was blocked in skim milk for $4 \mathrm{~h}$ and incubated with primary antibody for $12 \mathrm{~h}$ at $4^{\circ} \mathrm{C}$. Then the membrane was incubated with secondary antibody for $2 \mathrm{~h}$ at $37^{\circ} \mathrm{C}$. The chemiluminescence intensity was detected using an ECL kit (Beyotime). The primary antibodies p21 (1/1500; ab218311), CyclinD1 (1/1000; ab40754), B-cell lymphoma-2 (Bcl-2; 1/1000; ab32124), cleaved caspase 3 (cleavedcasp-3, 1/500; ab32042), E-cadherin (ab40772, 1/2000), N-cadherin (ab18203, 1/2000), Vimentin (ab45939, 1/1000), ATAD2 (1/500; ab176319), GAPDH (1/2500; ab9485) and secondary antibody $(1 / 10,000 ;$ ab97051) were purchased from Abcam (Cambridge, MA, USA).

\section{Transwell assay}

Transwell chambers (Solarbio) were utilized to monitor the migrated and invaded abilities of Y79 and WERI-Rb-1 cells. For the migration assay, RPMI-1640 medium containing 10\% FBS was supplemented 
into the lower chamber, while the upper chamber was added with suspended cells in serum-free medium. After 24 -h incubation, the migrated cells were stained with $0.1 \%$ crystal violet. Cell numbers in 10 random fields were counted using a light microscope and calculated using Image Pro Plus. The protocol of invasion assay was similar to that of the migration assay, while the difference was that the upper chamber was covered with a matrigel matrix (BD, Franklin Lakes, USA).

\section{Dual-luciferase reporter assay}

The wide type and mutant complementary sequences of MALAT1 or 3'-untranslated regions (3'UTR) of ATAD2 were inserted into pGL3 vector (Promega, Madison, WI, USA) to construct the luciferase reporter, named as WT-MALAT1, MUT-MALAT1, WT-ATAD2 or MUT-ATAD2. The luciferase reporter and miR-655-3p or miR-con were co-transfected into Y79 and WERI-Rb-1 cells. Following 48-h incubation, the luciferase activity was evaluated using Dual-Lucy Assay Kit (Solarbio).

\section{Mice model experiment}

The animal experiment was carried out according to the protocol approved by the Animal Care Committee of Weihai Central Hospital. The four-week-old nude mice were bought from Shanghai Laboratory Animal Company (SLAC, Shanghai, China) and divided into two groups ( $n=6$ per group): sh-MALAT1 group and sh-con group. The Y79 cells $\left(4 \times 10^{6}\right)$ transfected with sh-MALAT1 or sh-con were inoculated into nude mice. The tumor volume was measured every 7 days continued to 28 days and calculated according to the formula: volume $\left(\mathrm{mm}^{3}\right)=$ width $^{2} \times$ length $/ 2$. Then the xenograft tumors were resected, the tumors' weight was tested and the tumors were stored in $-80^{\circ} \mathrm{C}$ refrigerator until the further exploration.

\section{Statistical analysis}

GraphPad Prism 7 (GraphPad Inc., La Jolla, CA, USA) was used to process the experimental data which were repeated for at least three times. The Student's $t$-test was used to compare the data between the two groups, and one-way analysis of variance (ANOVA) was utilized to analyze the comparison among multiple groups. $P<0.05$ was considered statistically different.

\section{Results}

\section{MALAT1 was significantly up-regulated and miR-655-3p was remarkably down-regulated in RB}

Firstly, we measured the levels of MALAT1 and miR-655-3p in thirty RB tissues and eighteen normal globe tissues. As presented in Fig. 1A, the level of MALAT1 was apparently elevated in RB tissues compared to that in normal tissues. Also, the level of miR-655-3p was distinctly decreased in RB tissues (Fig. 1B). Besides, the scatter diagram exhibited that the level of miR-655-3p in RB was negatively linear correlated with the level of MALAT1 (Fig. 1C). These data indicated that MALAT1 was highly expressed, while miR655-3p was lowly expressed in RB. 
MALAT1 silencing constrained cell proliferation, metastasis, and EMT but promoted apoptosis in Y79 and WERI-Rb-1 cells

In order to investigate the functions of MALAT1 in RB, we firstly measured the level of MALAT1 in RB cells. The results displayed that MALAT1 was obviously increased in RB cell lines (Y79 and WERI-Rb-1) in contrast to that in retinal pigment epithelial cell line ARPE19 (Fig. 2A). The qRT-PCR results confirmed the knockdown efficiency, indicated by the dramatical reduction of MALAT1 in Y79 and WERI-Rb-1 cells transfected with si-MALAT1 (Fig. 2B). Moreover, the transfection of si-MALAT1 resulted in the marked decline of cell viability in Y79 and WERI-Rb-1 cells in comparison with that in si-con group (Fig. 2C-D). While the apoptotic rate was notably facilitated in Y79 and WERI-Rb-1 cells transfected with si-MALAT1 (Fig. 2E). Since p21, CyclinD1 as proliferation-related factors and Bcl-2, cleaved-casp-3 as apoptosisassociated markers, the protein levels of them were tested in Y79 and WERI-Rb-1 cells. The Western blotassay exhibited that the protein levels of p21 and cleaved-casp-3 were strikingly accelerated, but the protein levels of CyclinD1, Bcl-2 were conspicuously decreased in si-MALAT1-transfected siY79 and WERIRb-1 cells (Fig. 2F-G). Besides, the introduction of si-MALAT1 notably reduced the migrated and invaded abilities of $\mathrm{Y} 79$ and WERI-Rb-1 cells compared with that in si-con group (Fig. $2 \mathrm{H}-\mathrm{I}$ ). Given that E-cadherin, $\mathrm{N}$-cadherin, and Vimentin as epithelial-mesenchymal transition (EMT) protein markers, the levels of these protein were detected. As exhibited in Fig. 2J-K, the protein level of E-cadherin was apparently elevated, while N-cadherin and Vimentin were strikingly decreased in si-MALAT1 group in comparison with that in si-con group. Taken together, the depletion of MALAT1 inhibited cell proliferation, migration, invasion, and EMT but facilitated apoptosis in Y79 and WERI-Rb-1 cells.

\section{MiR-655-3p overexpression suppressed cell proliferation, migration, invasion, and EMT while boosted apoptosis in Y79 and WERI-Rb-1 cells}

Meanwhile, the level of miR-655-3p was evidently down-regulated in Y79 and WERI-Rb-1 cells (Fig. 3A). The level of miR-655-3p was extremely enhanced in $Y 79$ and WERI-Rb-1 cells transfected with miR-655-3p mimics, affirming the transfection efficiency (Fig. 3B). Furthermore, CCK8 assay indicated that cell viability, migrated and invaded abilitieswere significantly declined in Y79 and WERI-Rb-1 cells transfected with miR-655-3p related to that in miR-con group (Fig. 3C-D and H-I). The transfection of miR-655-3p greatly augmented apoptotic rate compared to that in miR-con group (Fig .3E). As shown in Fig. 3F-G, the introduction of miR-655-3p apparently expedited the protein levels of p21, cleaved-casp-3 but drastically reduced the protein levels of CyclinD1, Bcl-2 in Y79 and WERI-Rb-1 cells. Besides, the transfection of miR655-3p contributed to the distinctly down-regulation of the level of E-cadherin protein, as well as the remarkably decrease of the protein levels of N-cadherin and Vimentin in miR-655-3p group related to that in miR-con group (Fig. 3J-K). To sum up, these results disclosed that the overexpression of miR-655-3p retarded RB progression.

\section{MALAT1 negatively interacted with miR-655-3p in Y79 and WERI-Rb-1 cells}

To explore the mechanism of MALAT1 in RB, starBase online database (http://starbase.sysu.edu.cn/starbase2/) was used to predict the putative targets of MALAT1. The results 
showed that miR-655-3p was a candidate of MALAT1 (Fig. 4A). Following dual-luciferase reporter assay demonstrated that the transfection of miR-655-3p contributed to the remarkable reduction of luciferase activity of WT-MALAT1 reporter related to that in miR-con group, while the luciferase activity of MUTMALAT1 reporter had no change (Fig. 4B-C). In addition, the level of miR-655-3p was enhanced in siMALAT1-transfected Y79 and WERI-Rb-1 cells, while decreased in pcDNA-MALAT1 group (Fig. 4D-E). These data implicated that MALAT1 sponged miR-655-3p in Y79 and WERI-Rb-1 cells.

\section{MALAT1 knockdown inhibited cell proliferation but facilitated apoptosis in RB by sponging miR-655-3p}

To explore the mechanism and functions of MALAT1 and miR-655-3p in RB, si-MALAT1 and anti-miR-con were transfected into Y79 and WERI-Rb-1 cells. . As exhibited in Fig. 5A, the level of miR-655-3p was distinctly increased in si-MALAT1-transfected Y79 and WERI-Rb-1 cells, while obviously declined by the introduction of anti-miR-655-3p. Moreover, the emergence of anti-miR-655-3p relieved the inhibitory effects on cell viability and the abilities of migrated and invaded of $Y 79$ and WERI-Rb- 1 cells inhibited by si-MALAT1 (Fig. 5B-C and G-H). MiR-655-3p inhibitor attenuated the promoted impact on apoptotic rate in Y79 and WERI-Rb-1 cells induced by MALAT1 silencing (Fig.5D). In addition, the transfection of miR-655$3 p$ inhibitor counteracted the facilitated effect on the protein levels of $p 21$, cleaved-casp-3, and Ecadherin, as well as the restraint effect on the protein levels of CyclinD1, Bcl-2, N-cadherin, and Vimentin in Y79 and WERI-Rb-1 cells caused by si-MALAT1 (Fig. 5E-F and I-J). These data implicated that the silencing of MALAT1 suppressed RB progression by sponging miR-655-3p.

\section{ATAD was a candidate target of miR-655-3p in Y79 and WERI-Rb-1 cells}

In order to explore the mechanism of miR-655-3p in RB, starBase online database was used to search the putative target of miR-655-3p in RB. As shown in Fig. 6A, ATAD2 3'UTR had complementary binding sites with miR-655-3p. The luciferase activity of WT-ATAD2 reporter was markedly declined in Y79 and WERI$\mathrm{Rb}-1$ cells transfected with miR-655-3p, while MUT-ATAD2 reporter's activity had no apparent fluctuation in any group (Fig. 6B-C). Besides, the mRNA and protein levels of ATAD2 were highly expressed in RB tissues and cells (Fig. 6D-G). Also, the level of ATAD2 was negatively correlated with miR-655-3p (Fig. $6 \mathrm{H})$. The qRT-PCR and Western blot assay showed that the mRNA and protein levels of ATAD2 were distinctly reduced in miR-655-3p-transfected $\mathrm{Y} 79$ and WERI-Rb-1 cells, while dramatically enhanced in Y79 and WERI-Rb-1 cells transfected with anti-miR-65-3p (Fig. 6I-L). These data demonstrated that ATAD21 was negatively interacted with miR-655-3p in Y79 and WERI-Rb-1 cells.

\section{MiR-655-3p negatively regulated ATAD2 expression to impede cell proliferation and impel apoptosis in Y79 and WERI-Rb-1 cells}

To investigate whether the effects of miR-655-3p on RB progression were mediated by ATAD2, the restoration experiments of ATAD2 was conducted. As shown in Fig. 7A-C, the mRNA and protein levels of ATAD2 were remarkably down-regulated in Y79 and WERI-Rb-1 cells transfected with miR-655-3p, while relieved in miR-655-3p and pcDNA-ATAD2 group Furthermore, the transfection of pcDNA-ATAD2 receded the repressive impacts on cell viability and migrated and invaded abilities retarded by miR-655-3p in Y79 
and WERI-Rb-1 cells (Fig. 7D-E). The apoptotic rate was distinctly enhanced in Y79 and WERI-Rb-1 cells transfected with miR-655-3p, while partly attenuated in Y79 and WERI-Rb-1 cells co-transfected with miR655-3p and pcDNA-ATAD2 (Fig. 7F). Finally, the transfection of pcDNA-ATAD2 mitigated the accelerated effect on the protein levels of p21, cleaved-casp-3, E-cadherin as well as the restraint effect on the protein levels of CyclinD1, Bcl-2, N-cadherin, and Vimentin in Y79 and WERI-Rb-1 cells caused by miR-655-3p mimics (Fig. 7G-H and K-L). These data implicated that miR-655-3p hampered cell proliferation, metastasis, and EMT while impelled apoptosis in Y79 and WERI-Rb-1 cells modulating ATAD2.

\section{MALAT1 positively regulated ATAD2 expression to accelerate cell proliferation but block apoptosis in RB by sponging miR-655-3p}

To further explore the relationship among MALAT1, miR-655-3p and ATAD2 in RB, we detected the protein level of ATAD2 in Y79 and WERI-Rb-1 cells transfected with si-con, si-MALAT1, si-MALAT1 + anti-miR-con, or si-MALAT1 + anti-miR-655-3p. As presented in Fig. 8A-C, the level of ATAD2 mRNA and protein were apparently down-regulated in $\mathrm{Y} 79$ and WERI-Rb-1 cells transfected with si-MALAT1, while regained by the introduction of anti-miR-655-3p. Also, the scatter plot showed that the level of MALAT1 was positively linear correlated with ATAD2 (Fig. 8D). Meanwhile, the introduction of si-MALAT1 effectively downregulated the levels of mRNA and protein in $Y 79$ and WERI-Rb-1 cells, while reversed by the emergence of pcDNA-ATAD2 (Fig. 8E-G). Moreover, the transfection of pcDNA-ATAD2 weakened the suppressive impacts on cell viability, migrated and invaded abilitiesin Y79 and WERI-Rb-1 cells inhibited by si-MALAT1 (Fig.8H-I and M-N). Whereas, the apoptotic rate showed the opposite trend. Briefly, the apoptotic rate was obviously increased in Y79 and WERI-Rb-1 cells transfected with si-MALAT1, while partly attenuated in Y79 and WERI-Rb-1 cells co-transfected with si-MALAT1 and pcDNA-ATAD2 (Fig. 8J). The Western blot assay indicated that the emergence of pcDNA-ATAD2 neutralized the promoted effects on the protein levels of p21, cleaved-casp-3, and E-cadherin, as well as the inhibitory effects on the protein levels of CyclinD1, Bcl-2, N-cadherin, and Vimentin in Y79 and WERI-Rb-1 cells caused by MALAT1 silencing (Fig.8K-L and O-P). These data demonstrated that MALAT1 positively modulated ATAD2 expression to regulate cell behaviors in Y79 and WERI-Rb-1 cells by sponging miR-655-3p.

\section{MALAT1 depletion impeded xenograft tumor growth in vivo}

To further verify the effects of MALAT1 in vivo, the mice model experiments were constructed. Firstly, we affirmed sh-MALAT1 or sh-con transfection efficiency, indicated by the striking down-regulation of MALAT1 in Y79 cells (Fig. 9A). The measurement results presented that the weight and volume were both substantially declined in sh-MALAT1 group related to that in sh-NC group (Fig. 9B-C). In addition, the level of miR-655-3p was significantly elevated in sh-MALAT1 group, while the mRNA and protein levels of ATAD2 were notably reduced in sh-MALAT1 group (Fig. 9D-E). These results suggested that the silencing of MALAT1 blocked xenograft tumor growth in vivo.

\section{Discussion}


It is well known that tumor progression is a complicated issue for patients who suffered the pain of the tumor. RB is a rare type of intraocular cancer. Convincing evidence indicated that MALAT1 was an oncogene in diverse types of cancers. In the current study, the roles of MALAT1 in RB were mainly explored. The results suggested that MALAT1 promoted RB progression partially through miR-6553p/ATAD2 axis.

Emerging evidence demonstrated that the dysregulation of MALAT1 was implicated in tumor progression. For instance, a study in ovarian cancer disclosed that MALAT1 was strikingly elevated in ovarian cancer, while its depletion retarded cell growth, metastasis in vitro [8]. Another study in gastric cancer (GC) revealed that MALAT1 was conspicuously boosted in GC; but its depletion impeded cell growth, metastasis in vitro, as well as induced apoptosis [9]. The similar results were also reported in colorectal cancer [10], papillary thyroid cancer [11], and non-small cell lung cancer [12]. In this study, MALAT1 was highly expressed in RB. Meanwhile, MALAT knockdown blocked cell growth, metastasis, and EMT but enhanced apoptosis. In addition, the silencing of MALAT1 restrained xenograft tumor growth in vivo. These data also were consistent with previous reports in RB $[22,23]$. These data demonstrated that MALAT1 played vital roles in RB progression.

Currently, the aberrant expression of miR-655-3p was reported to associate with the behaviors of tumor cells. For instance, Wang et al. reported that miR-655-3p was low expressed in non-small cell lung cancer, miR-655-3p mimics resulted in the impediment of cell metastasis [18]. Another study in hepatocellular carcinoma (HCC) demonstrated that the low expression of miR-655-3p in HCC promoted cell growth, metastasis [24]. In this research, we found that miR-655-3p was down-regulated in RB, and MALAT1 was identified to sponge miR-655-3p. MALAT1 silencing curbed cell growth, mobility, and EMTwhile facilitated apoptosis by regulating miR-655-3p. Although miR-655-3p was not documented in RB, miR-655 was reported to be lowly expressed in RB and its overexpression impeded cell growth, metastasis while induced apoptosis by targeting PAX6 in vitro [25]. These data implicated that MALAT accelerated RB progression by sponging miR-655-3p.

Accumulating evidence demonstrated that ATAD2 was involved in the progression of tumors. For example, a research in cervical cancer reported that the mRNA and protein levels of ATAD2 were elevated in cervical cancer, and the knockdown of ATAD2 impeded cell growth, metastasis [21]. Another study in HCC illustrated that ATAD2 was enhanced in HCC, and its knockdown regulated cell behaviors mediated by miR-372 [26]. In this research, ATAD2 was verified as a candidate of miR-655-3p. Also, ATAD2 was upregulated in RB. MiR-655-3p affected cell behaviors by negatively regulating ATAD2. The similar results of ATAD2 in RB were in line with previous study [20]. MALAT1 knockdown down-regulated ATAD2 expression by sponging miR-655-3p. Furthermore, MALAT1 depletion decreased ATAD2 expression to regulate cell behaviors in RB cells. These results manifested that MALAT1 modulated ATAD2 to promote RB progression via miR-655-3p.

Taken together, we concluded that MALAT1, ATAD2 were up-regulated and miR-655-3p was downregulated in RB. MALAT1 positively regulated ATAD2 expression to regulate cell proliferation, megration, 
invasion, apoptosis, and EMT in RB by sponging miR-655-3p. The new regulatory network may shed light on the mechanism of RB progression. There were evidences indicated that MALAT1 promoted tumor progression through phosphatidylinositol-3-kinase (PI3K)/AKT/mammalian target of rapamycin (mTOR) signaling pathway in non-small cell lung cancer [27] and osteosarcoma [28]. In the next exploration, we will focus on whether the MALAT1/miR-655-3p/ATAD2-induced RB progression was mediated by $\mathrm{PI} 3 \mathrm{~K} / \mathrm{AKT} / \mathrm{mTOR}$ signaling pathway.

\section{Declarations}

\section{Acknowledgements}

Not applicable.

\section{Authors' contributions}

Yuxin Zhao and Zhaoxia Wang conceived, designed and revised the current study. Meili Gao, Xia Tian and Xuehong Wang analyzed the data. Hui Feng, Yuxin Zhao and Yuanyuan Cui wrote the manuscript. All authors read and approved the final manuscript.

\section{Funding}

Not applicable

\section{Availability of data and materials}

The datasets used and/or analyzed during the current study are available from the corresponding author on reasonable request.

\section{Ethics approval and consent to participate}

This study was conducted in accordance with the declaration of Helsinki. This study was conducted with approval from the Ethics Committee of Weihai Central Hospital. A written informed consent was obtained from all participants.

\section{Consent for publication}

Not applicable.

\section{Competing interests}

The authors declare that they have no competing interests.

\section{References}


1. Dimaras H, Corson TW. Retinoblastoma, the visible CNS tumor: A review. J Neurosci Res. 2019;97:2944.

2. Broaddus E, Topham A, Singh AD. Incidence of retinoblastoma in the USA: 1975-2004. Brit J Ophthalmol. 2009;93:21-23.

3. Lin P, O'Brien JM. Frontiers in the management of retinoblastoma. Am J Ophthalmol. 2009;148:192198.

4. Chantada GL, Qaddoumi I, Canturk S, Khetan V, Ma Z, Kimani K, et al. Strategies to manage retinoblastoma in developing countries. Pediatr Blood Cancer. 2011;56:341-348.

5. JT K, D C, JT L. Long noncoding RNAs: past, present, and future. Genetics. 2013;193:651-669.

6. Yan G, Su Y, Ma Z, Yu L, Chen N. Long noncoding RNA LINC00202 promotes tumor progression by sponging miR-3619-5p in retinoblastoma. Cell Struct Funct. 2019;44:51-60.

7. Shang Y. LncRNA THOR acts as a retinoblastoma promoter through enhancing the combination of cmyc mRNA and IGF2BP1 protein. Biomed Pharmacother. 2018;106:1243-1249.

8. Gordon MA, Babbs B, Cochrane DR, Bitler BG, Richer JK. The long non-coding RNA MALAT1 promotes ovarian cancer progression by regulating RBFOX2-mediated alternative splicing. Mol Carcinog. 2019;58:196-205.

9. Lee NK, Lee JH, Ivan C, Ling H, Zhang X, Park CH, et al. MALAT1 promoted invasiveness of gastric adenocarcinoma. BMC Cancer. 2017;17:46.

10. Xie J, Li W, Li X, Ye W, Shao C. LncRNA MALAT1 promotes colorectal cancer development by sponging miR-363-3p to regulate EZH2 expression. J Biol Regul Homeost Agents. 2019;33:331-343.

11. Liu J, Dong H, Yang Y, Qian Y, Liu J, Li Z, et al. Upregulation of long noncoding RNA MALAT1 in papillary thyroid cancer and its diagnostic value. Future Oncol. 2018;14:3015-3022.

12. Chen W, Zhao W, Chen S, Zhang L, Guo Z, Wang L, et al. Expression and correlation of MALAT1 and SOX9 in non-small cell lung cancer. Clin Respir. 2018;12:2284-2291.

13. Huang Y, Shen XJ, Zou Q, Wang SP, Tang SM, Zhang GZ. Biological functions of microRNAs: a review. J Physiol Biochem. 2011;67:129-139.

14. Zhang Y, Xue C, Zhu X, Zhu X, Xian H, Huang Z. Suppression of microRNA-125a-5p upregulates the TAZ-EGFR signaling pathway and promotes retinoblastoma proliferation. Cell Signal. 2016;28:850860 .

15. Sun Z, Zhang A, Zhang L. Inhibition of microRNA-492 attenuates cell proliferation and invasion in retinoblastoma via directly targeting LATS2. Mol Med Rep. 2019;19:1965-1971.

16. Li J, You X. MicroRNA-758 inhibits malignant progression of retinoblastoma by directly targeting PAX6. Oncol Rep. 2018;40:1777-1786.

17. Zha J, Chen D. MiR-655-3p inhibited proliferation and migration of ovarian cancer cells by targeting RAB1A. Eur Rev Med Pharmacol Sci. 2019;23:3627-3634.

18. Wang W, Cao R, Su W, Li Y, Yan H. miR-655-3p inhibits cell migration and invasion by targeting pituitary tumor-transforming 1 in non-small cell lung cancer. Biosci Biotechnol Biochem. 2019:1-6. 
19. Hussain M, Zhou Y, Song Y, Hameed HA, Jiang H, Tu Y, et al. ATAD2 in cancer: a pharmacologically challenging but tractable target. Expert Opin Ther Targets. 2018;22:85-96.

20. Wu S, Han M, Zhang C. Overexpression of microRNA-186 inhibits angiogenesis in retinoblastoma via the Hedgehog signaling pathway by targeting ATAD2. J Cell Physiol. 2019.

21. Zheng $L, L i T$, Zhang $Y$, Guo $Y$, Yao J, Dou $L$, et al. Oncogene ATAD2 promotes cell proliferation, invasion and migration in cervical cancer. Oncol Rep. 2015;33:2337-2344.

22. Liu S, Yan G, Zhang J, Yu L. Knockdown of long noncoding RNA (IncRNA) metastasis-associated lung adenocarcinoma transcript 1 (MALAT1) inhibits proliferation, migration, and invasion and promotes apoptosis by targeting miR-124 in retinoblastoma. Oncol Res. 2018;26:581-591.

23. Huang J, Yang Y, Fang F, Liu K. MALAT1 modulates the autophagy of retinoblastoma cell through miR-124-mediated stx17 regulation. J Cell Biochem. 2018;119:3853-3863.

24. Wu G, Zheng K, Xia S, Wang Y, Meng X, Qin X, et al. MicroRNA-655-3p functions as a tumor suppressor by regulating ADAM10 and $\beta$-catenin pathway in hepatocellular carcinoma. J Exp Clin Canc Res. 2016;35:89.

25. Zhang M, Li Q, Pan Y, Wang H, Liu G, Yin H. MicroRNA-655 attenuates the malignant biological behaviours of retinoblastoma cells by directly targeting PAX6 and suppressing the ERK and p38 MAPK signalling pathways. Oncol Rep. 2018;39:2040-2050.

26. Wu G, Liu H, He H, Wang Y, Lu X, Yu Y, et al. miR-372 down-regulates the oncogene ATAD2 to influence hepatocellular carcinoma proliferation and metastasis. BMC Cancer. 2014;14:107.

27. Tang Y, Xiao G, Chen Y, Deng Y. LncRNA MALAT1 promotes migration and invasion of non-small-cell lung cancer by targeting miR-206 and activating Akt/mTOR signaling. Anticancer Drugs. 2018;29:725-735.

28. Chen Y, Huang W, Sun W, Zheng B, Wang C, Luo Z, et al. LncRNA MALAT1 promotes Cancer metastasis in osteosarcoma via activation of the PI3K-Akt signaling pathway. Cell Physiol Biochem. 2018;51:1313-1326.

\section{Figures}
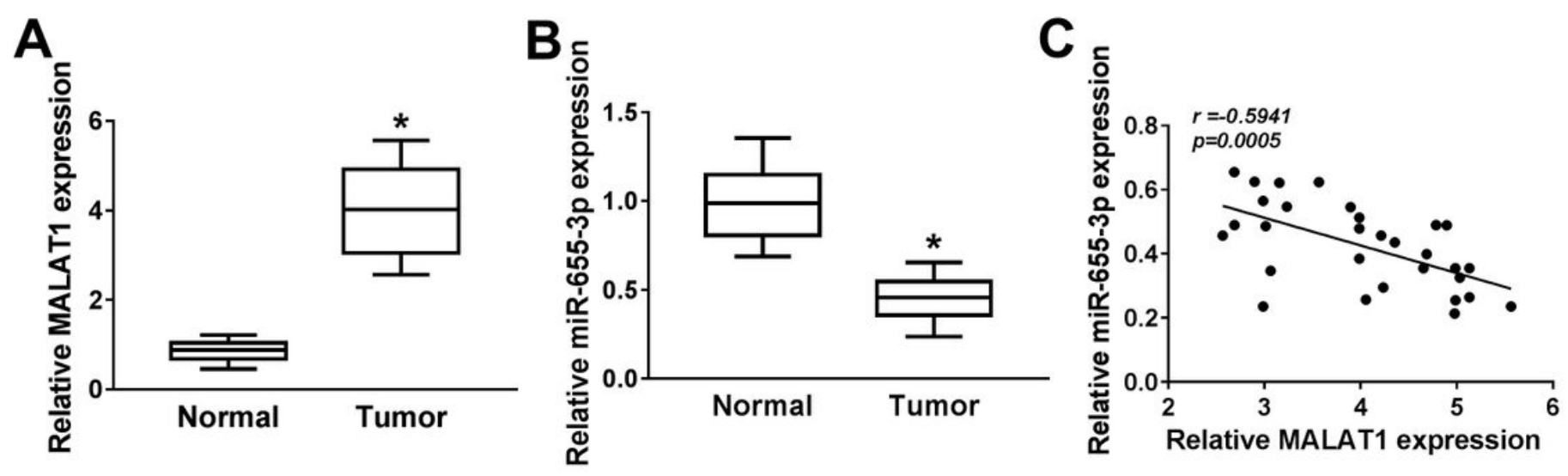
MALAT1 was significantly up-regulated and miR-655-3p was remarkably down-regulated in RB. (A-B) The levels of MALAT1 and miR-655-3p in RB tissues and normal globe tissues were tested by qRT-PCR. (C) The correlation between miR-655-3p and MALAT1 was verified by Pearson correlation analysis. ${ }^{*} \mathrm{P}<0.05$.
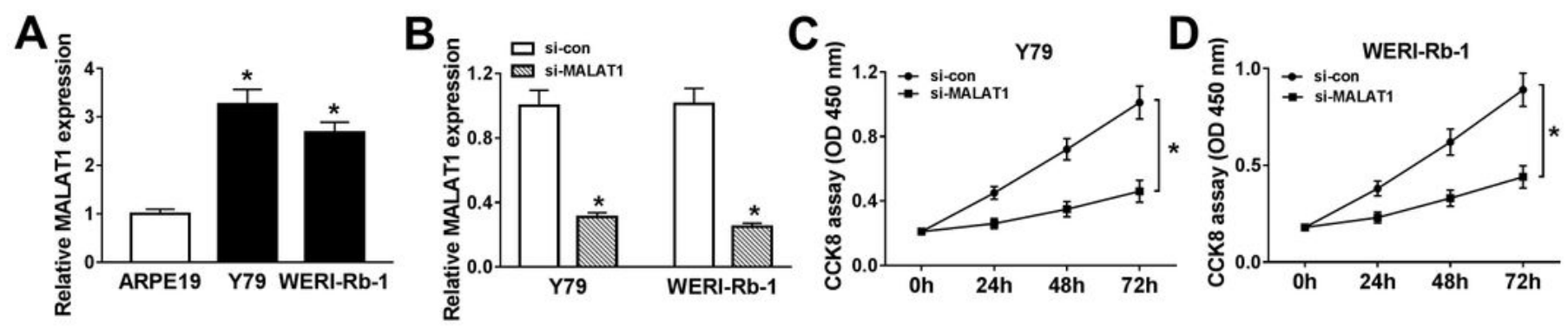

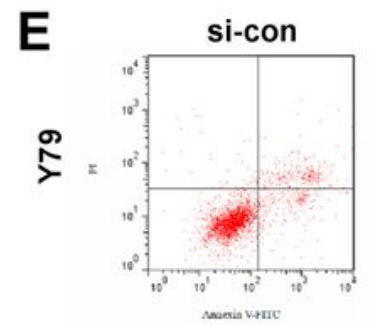

F
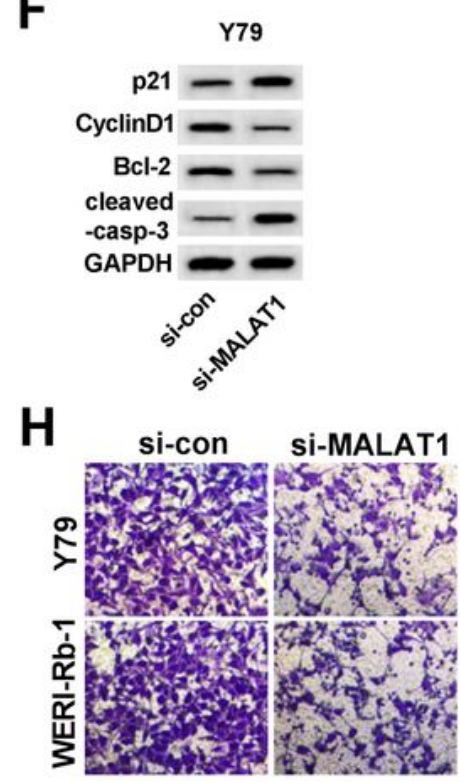

$\mathbf{J}$

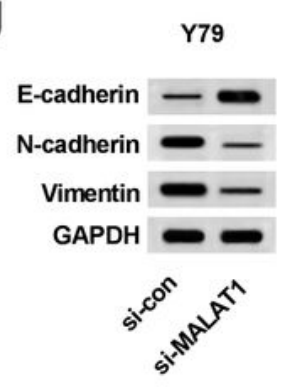

Si-MALAT1
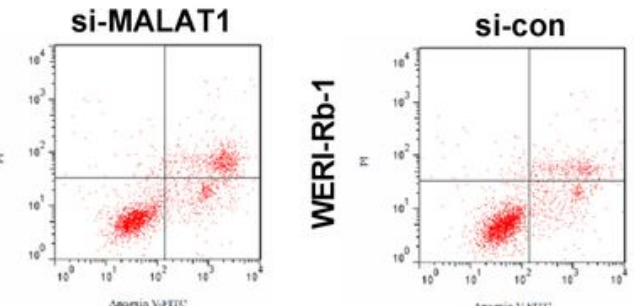

G
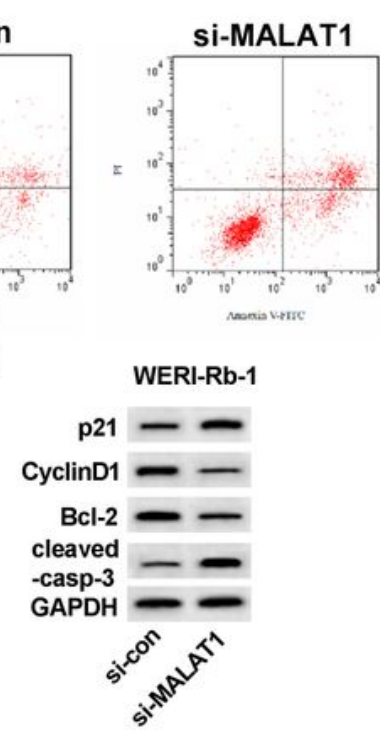

I

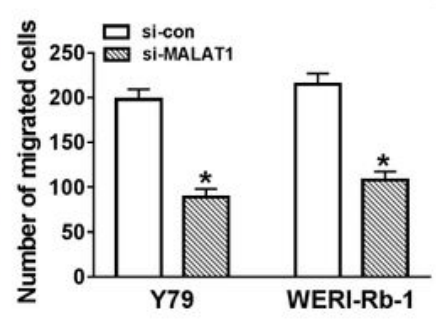

Y79

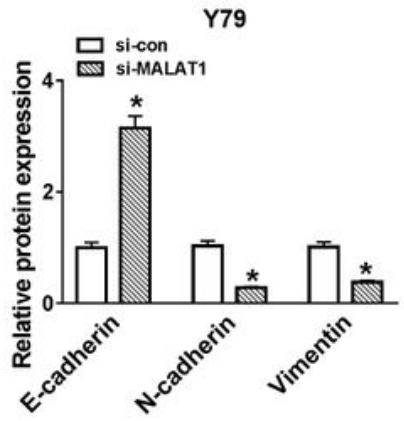

si-con Si-MALAT1

K

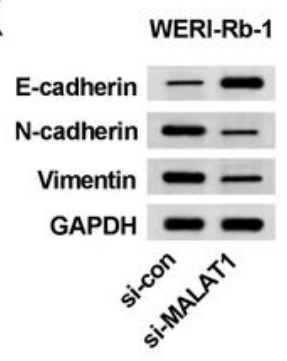

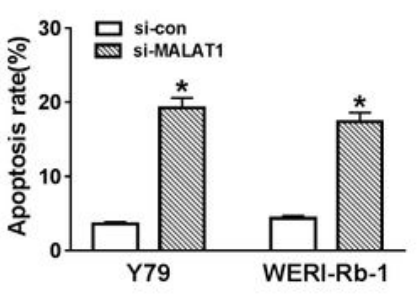
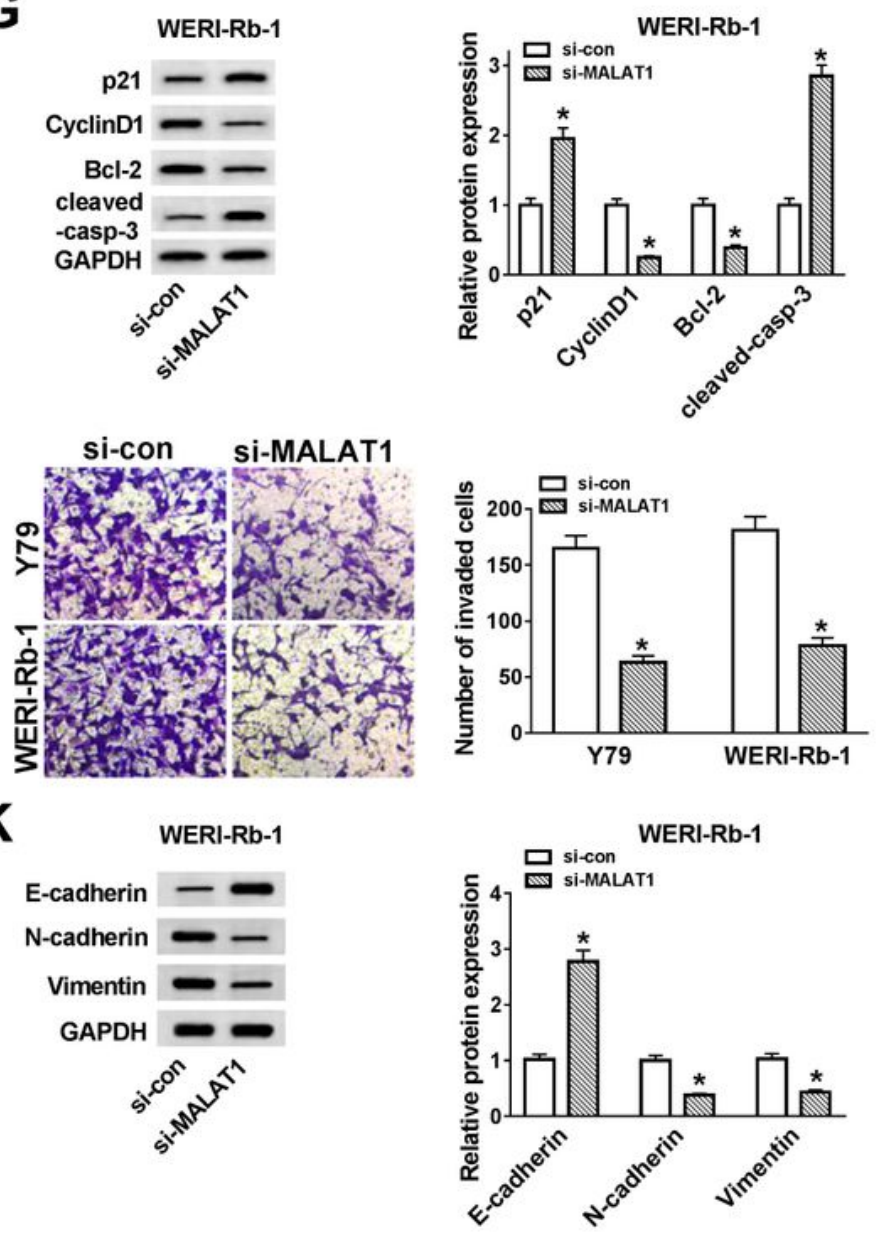

Figure 2 
MALAT1 silencing constrained cell proliferation but promoted apoptosis in $\mathrm{Y} 79$ and WERI-Rb-1 cells. (A) The level of MALAT1 in RB cells (Y79 and WERI-Rb-1) and retinal pigment epithelial cell ARPE19 was tested by qRT-PCR. (B) The level of MALAT1 in Y79 and WERI-Rb-1 cells transfected with si-MALAT1 or sicon was examined via qRT-PCR. (C-K) The Y79 and WERI-Rb-1 cells were transfected with si-con or siMALAT1. (C-D) The cell viability was monitored by CCK8 assay. (E) The apoptotic rate was assessed by flow cytometry. (F-G) The protein levels of p21, CyclinD1, Bcl-2, and cleaved-casp-3 were detected via Western blot assay. (H-I) The migrated and invaded abilities were tested by Transwell assay. (J-K) The protein levels of E-cadherin, $\mathrm{N}$-cadherin, and Vimentin were examined via Western blot assay. ${ }^{*} \mathrm{P}<0.05$.
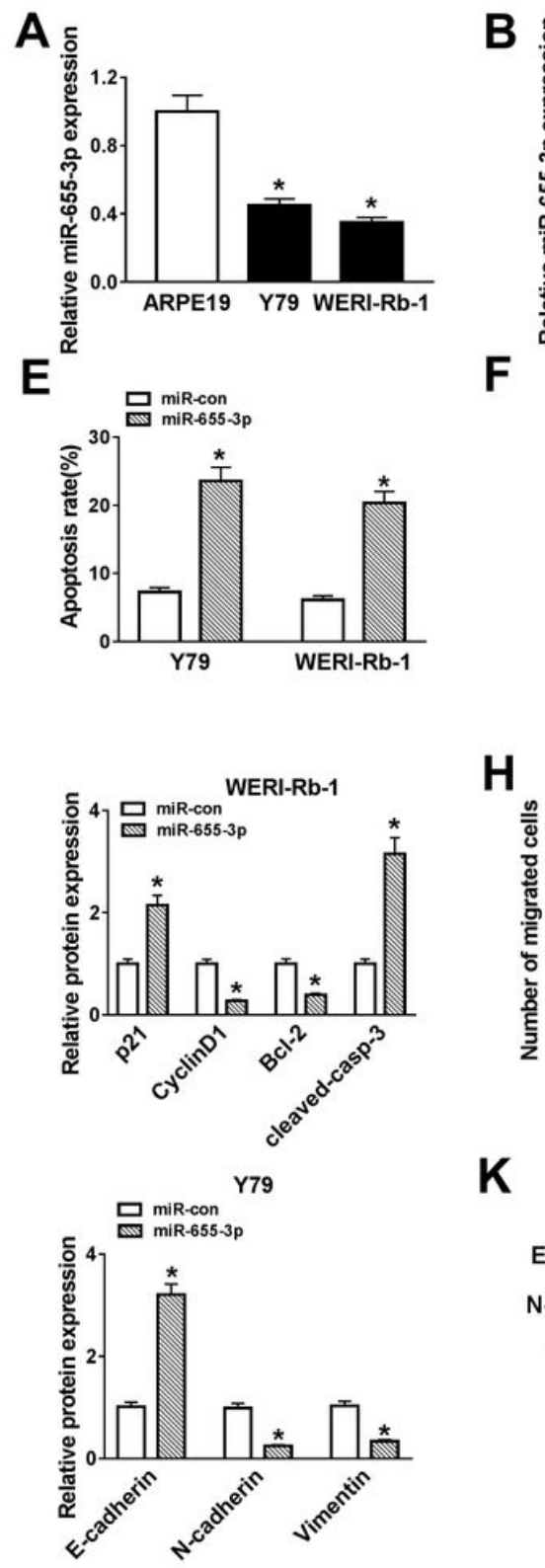

K
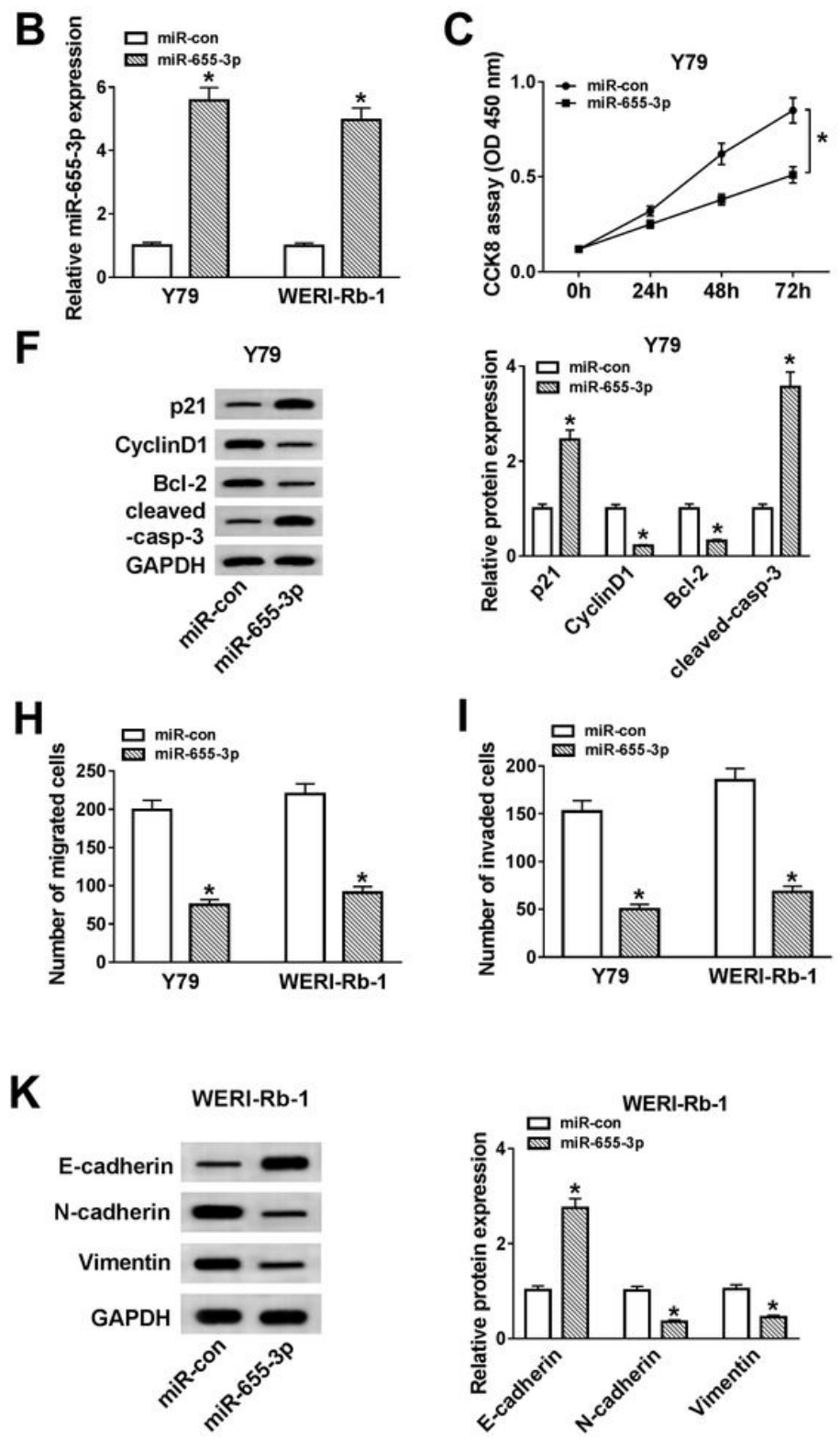

D

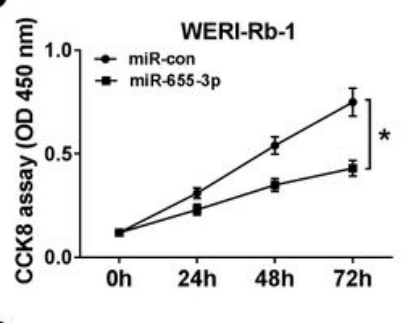

G
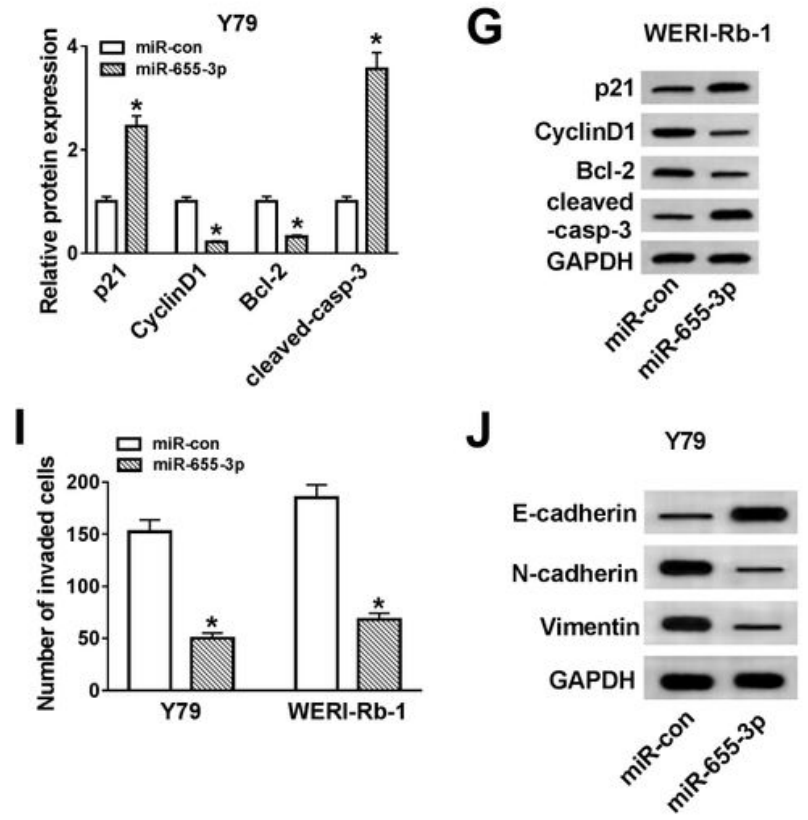

\section{Figure 3}

MiR-655-3p overexpression suppressed cell proliferation, migration, invasion, and EMT while boosted apoptosis in Y79 and WERI-Rb-1 cells. (A) The level of miR-655-3p in RB cells Y79 and WERI-Rb-1 and retinal pigment epithelial cell line ARPE19 was tested by qRT-PCR. (B-I) The Y79 and WERI-Rb-1 cells were 
transfected with miR-con or miR-655-3p. (B) The level of miR-655-3p was examined via qRT-PCR. (C-D) The cell viability was detected by CCK8 assay. (E) The apoptotic rate was assessed through flow cytometry. (F-G) The protein levels of p21, CyclinD1, Bcl-2, and cleaved-casp-3 were measured via Western blot assay. $(\mathrm{H}-\mathrm{I})$ The migrated and invaded abilities were evaluated by Transwell assay. (J-K) The protein levels of E-cadherin, $\mathrm{N}$-cadherin, and Vimentin were measured via Western blot assay. ${ }^{*} \mathrm{P}<0.05$.
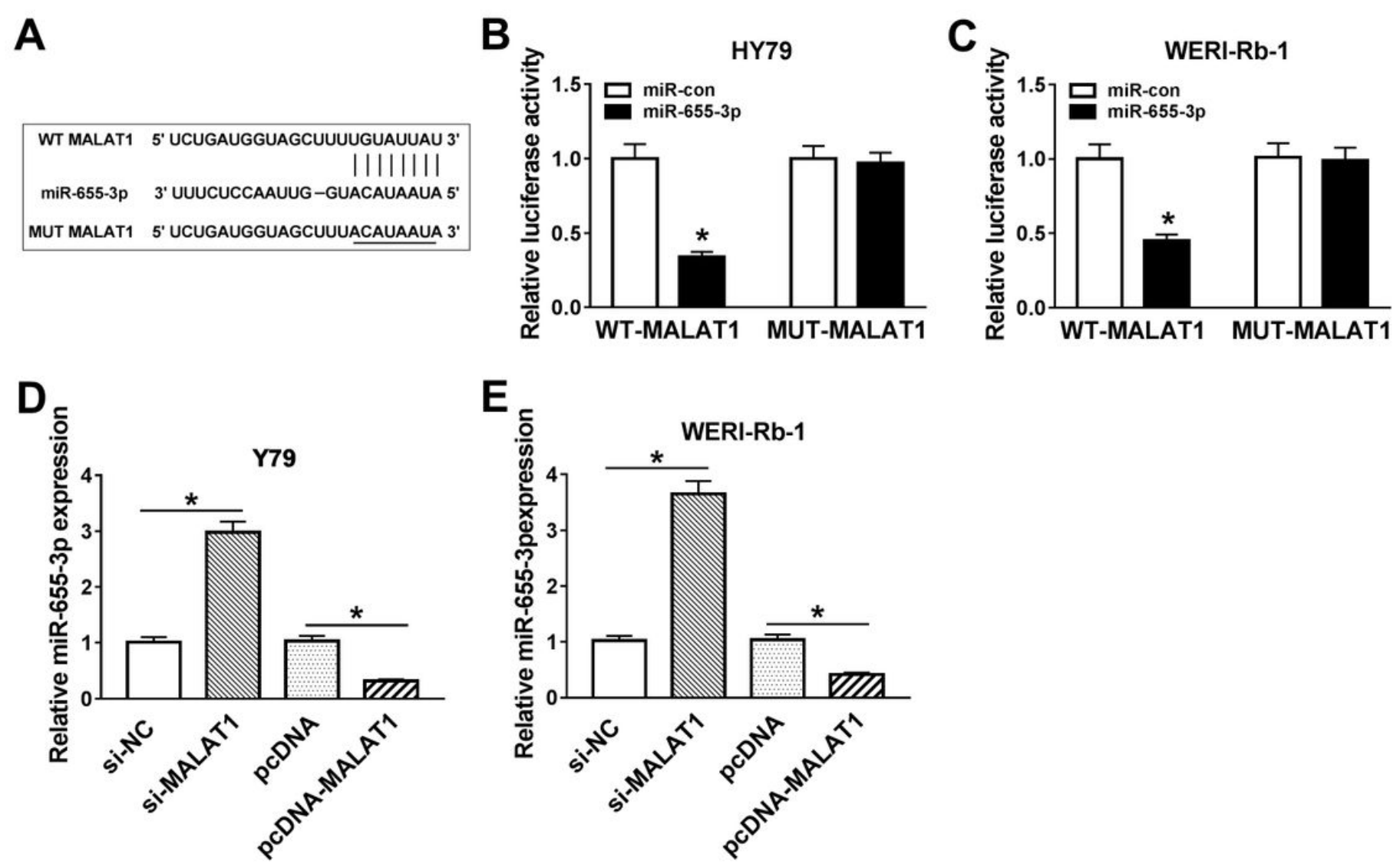

Figure 4

MALAT1 negatively interacted with miR-655-3p in Y79 and WERI-Rb-1 cells. (A) The complementary binding sites between MALAT1 and miR-655-3p were exhibited, as well as the mutant sequences of MALAT1. (B-C) The luciferase activities of WT-MALAT1 and MUT-MALAT1 reporter in Y79 and WERI-Rb-1 cells transfected with miR-con or miR-655-3p were evaluated by dual-luciferase reporter assay. (D-E) The level of miR-655-3p in Y79 and WERI-Rb-1 cells transfected with si-NC, si-MALAT1, pcDNA, or pcDNAMALAT1 was detected via qRT-PCR. *P<0.05. 


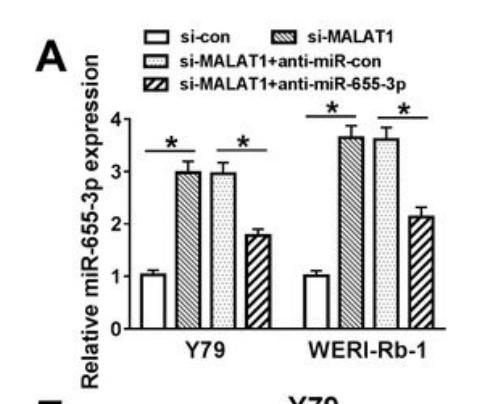

E
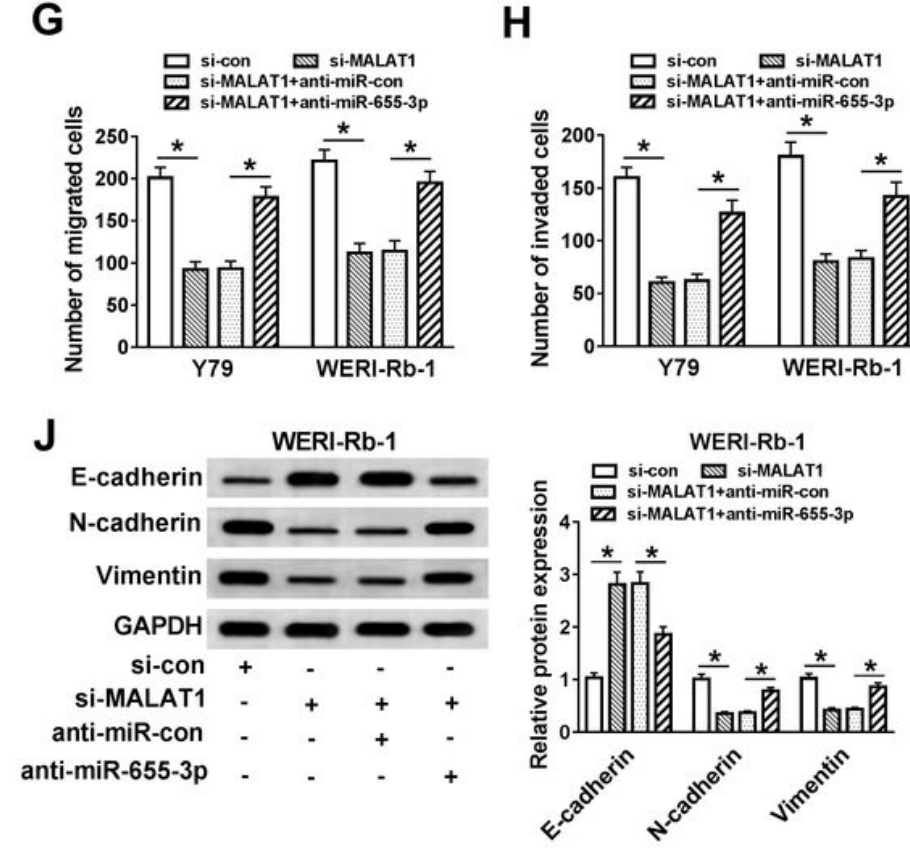

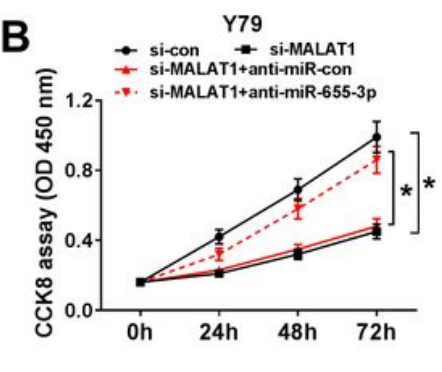

Y79

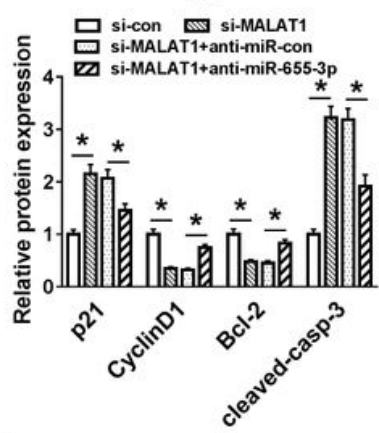

H
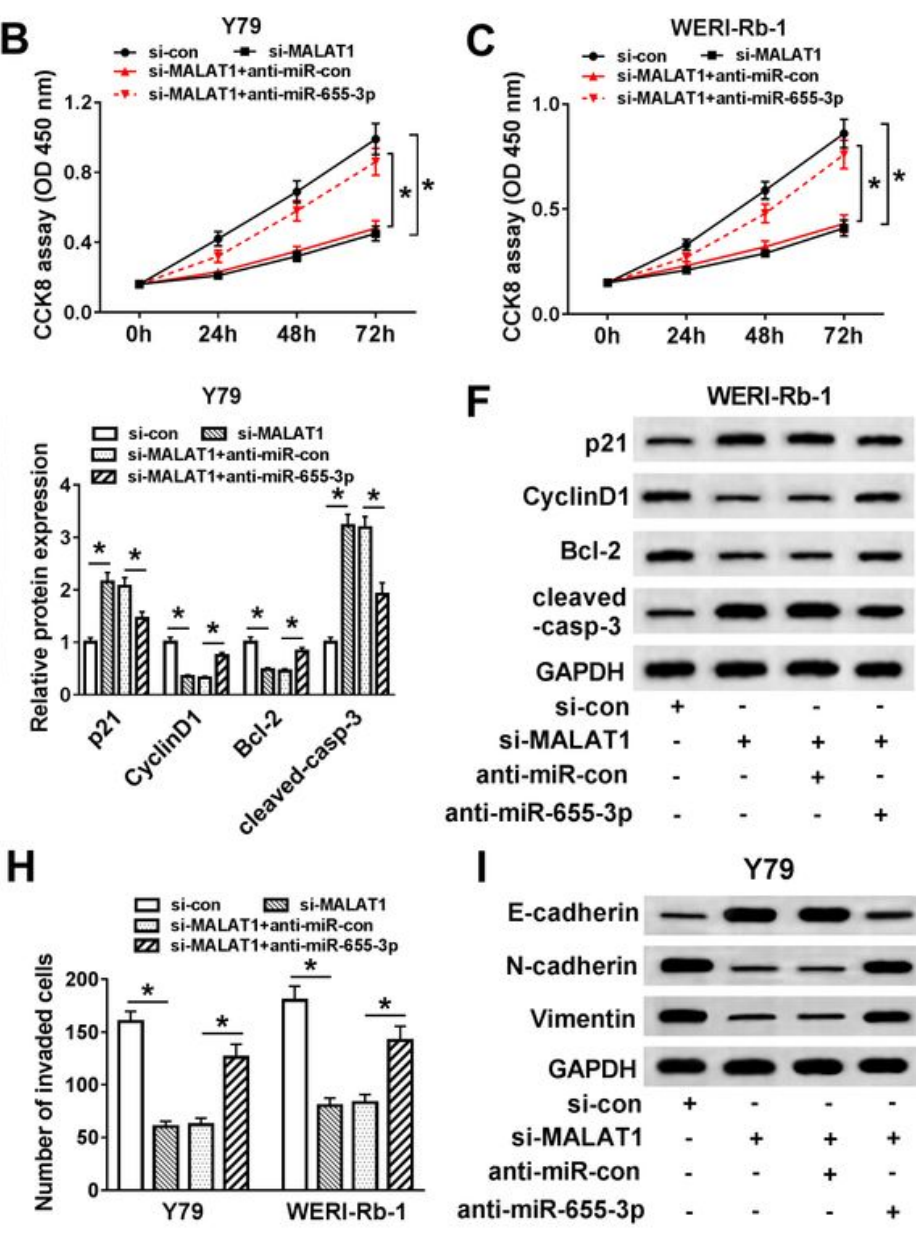

F

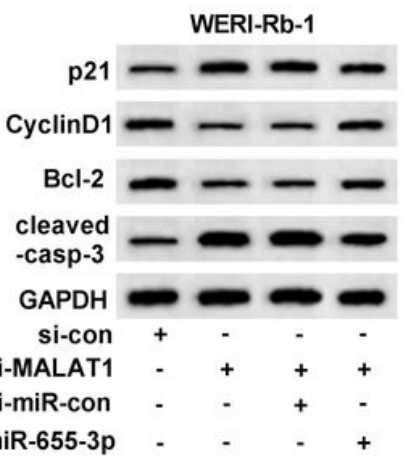

I

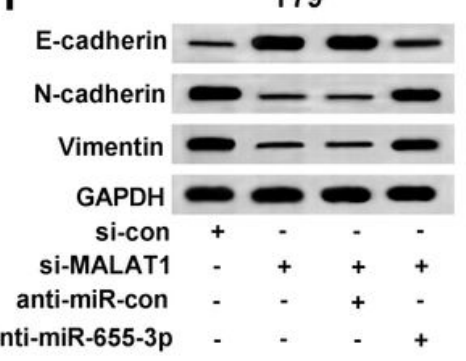

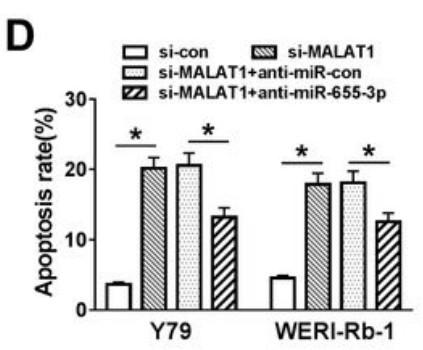

WERI-Rb-1
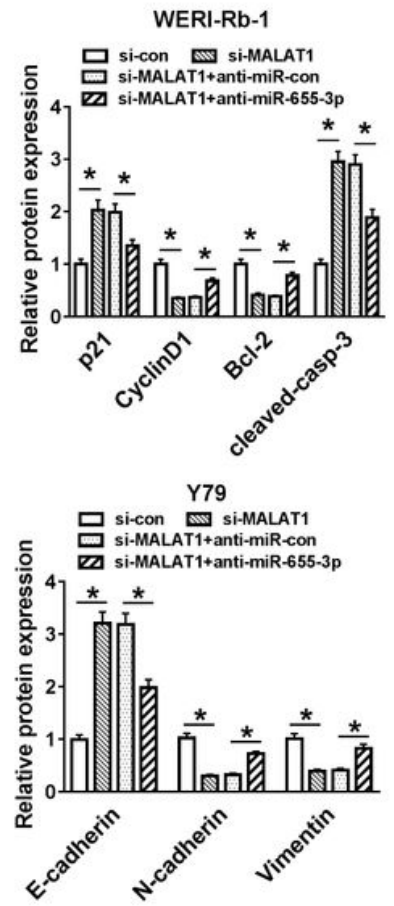

\section{Figure 5}

MALAT1 knockdown inhibited cell proliferation but facilitated apoptosis in RB by sponging miR-655-3p. (A-J) The Y79 and WERI-Rb-1 cells were transfected with si-con, si-MALAT1, si-MALAT1 + anti-miR-con or si-MALAT1 + anti-miR-655-3p. (A) The level of miR-655-3p was measured by qRT-PCR. (B-C) The cell viability was examined by CCK8 assay. (D) The apoptotic rate was evaluated through flow cytometry. (E-F and I-J) The protein levels of p21, CyclinD1, Bcl-2, cleaved-casp-3, E-cadherin, N-cadherin, and Vimentinwere tested via Western blot assay. (G-H) The migrated and invaded abilities were estimated by Transwell assay. ${ }^{\star} \mathrm{P}<0.05$. 

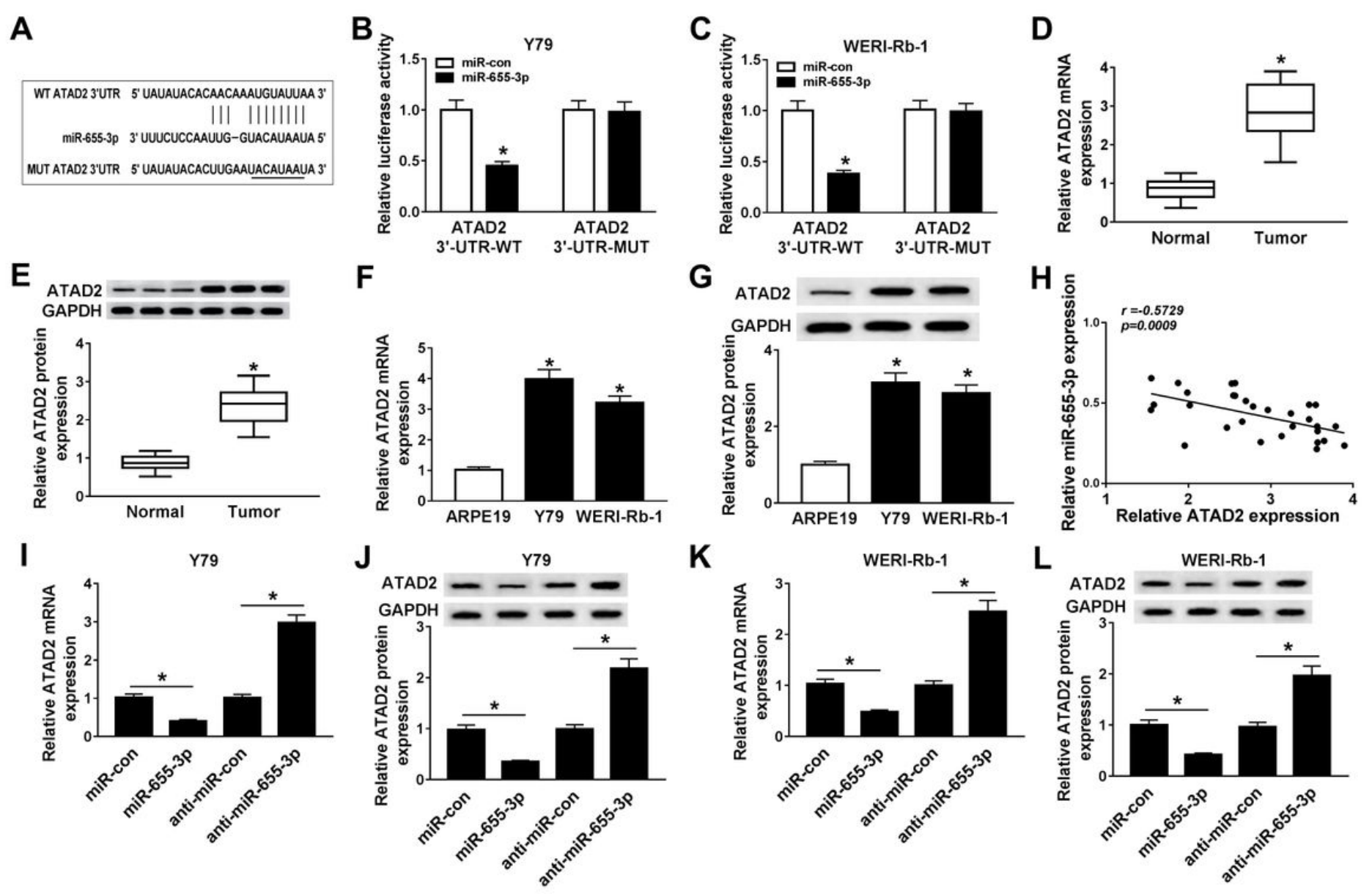

Figure 6

ATAD was a candidate target of miR-655-3p in Y79 and WERI-Rb-1 cells. (A) The complementary binding sites between miR-655-3p and ATAD2 3'UTR was exhibited, as well as the mutant sequences of ATAD2 3'UTR. (B-C) The luciferase activity of WT-ATAD2 or MUT-ATAD2 reporter in Y79 and WERI-Rb-1 cells transfected with miR-655-3p or miR-con was evaluated by dual-luciferase reporter assay. (D-G) The level of ATAD2 in RB tissues or cells was tested via qRT-PCR and Western blot assay, respectively. $(H)$ The correlation between miR-655-3p and ATAD2 was processed by Pearson test. (G-H) The mRNA and protein level of ATAD2 in Y79 and WERI-Rb-1 cells transfected with miR-con, miR-655-3p, anti-miR-con, anti-miR655-3p were examined via qRT-PCR and Western blot assay, respectively. ${ }^{*} P<0.05$.ATAD was a candidate target of miR-655-3p in Y79 and WERI-Rb-1 cells. (A) The complementary binding sites between miR-655$3 p$ and ATAD2 3'UTR was exhibited, as well as the mutant sequences of ATAD2 3'UTR. (B-C) The luciferase activity of WT-ATAD2 or MUT-ATAD2 reporter in Y79 and WERI-Rb-1 cells transfected with miR655-3p or miR-con was evaluated by dual-luciferase reporter assay. (D-G) The level of ATAD2 in RB tissues or cells was tested via qRT-PCR and Western blot assay, respectively. $(H)$ The correlation between miR-655-3p and ATAD2 was processed by Pearson test. (G-H) The mRNA and protein level of ATAD2 in Y79 and WERI-Rb-1 cells transfected with miR-con, miR-655-3p, anti-miR-con, anti-miR-655-3p were examined via qRT-PCR and Western blot assay, respectively. ${ }^{*}<0.05$. 
A

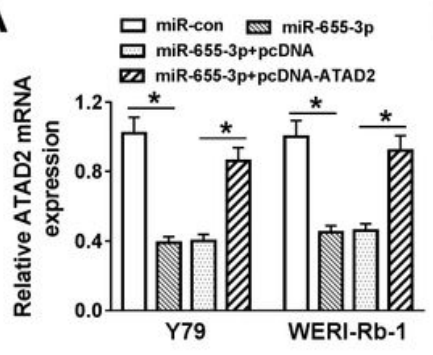

E

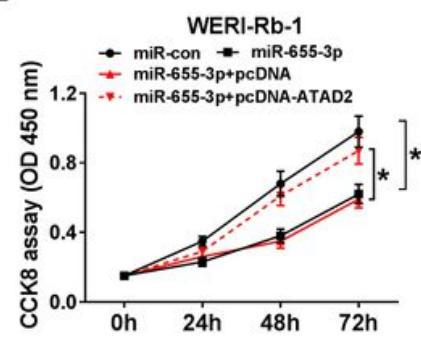

H

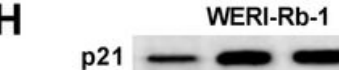

CyclinD1

$\mathrm{Bcl-2}$

cleaved

-casp-3

GAPDH

miR-con

miR-655-3p

pcDNA

pcDNA-ATAD2

K

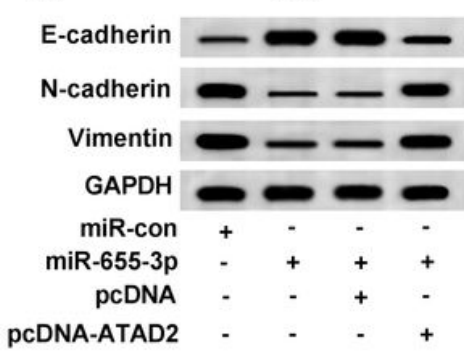

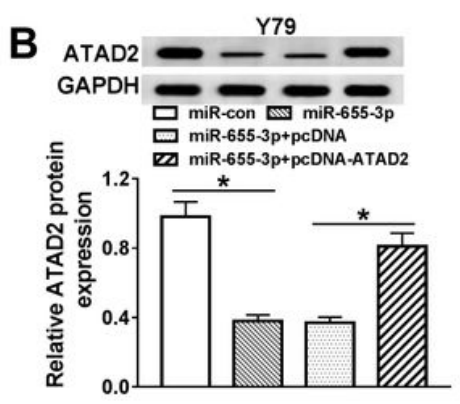

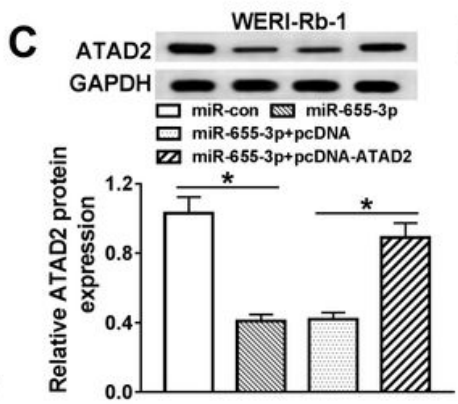

F

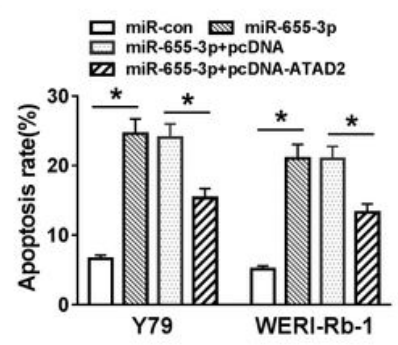

G
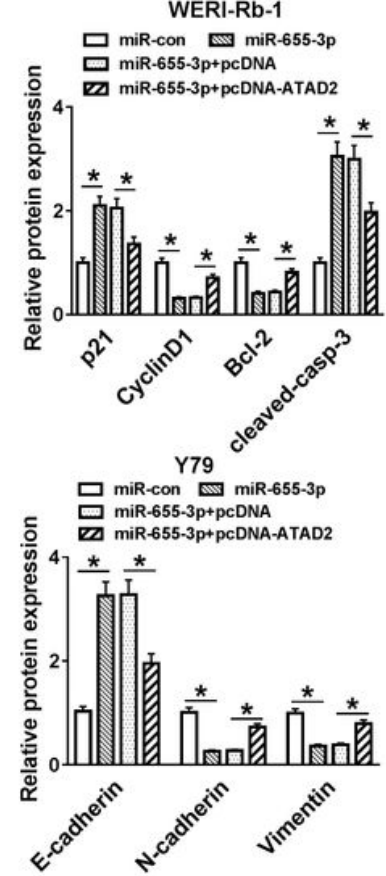

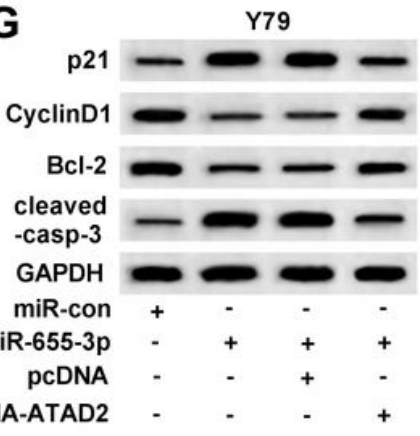

I

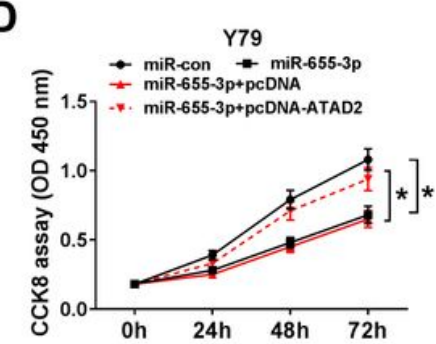

Y79

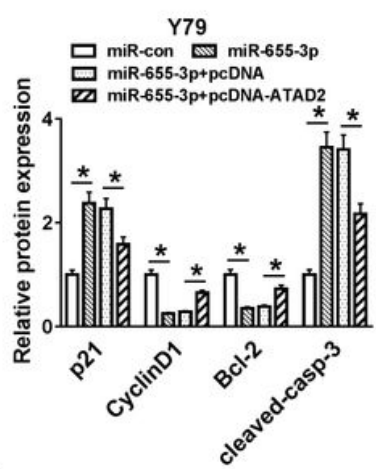

J
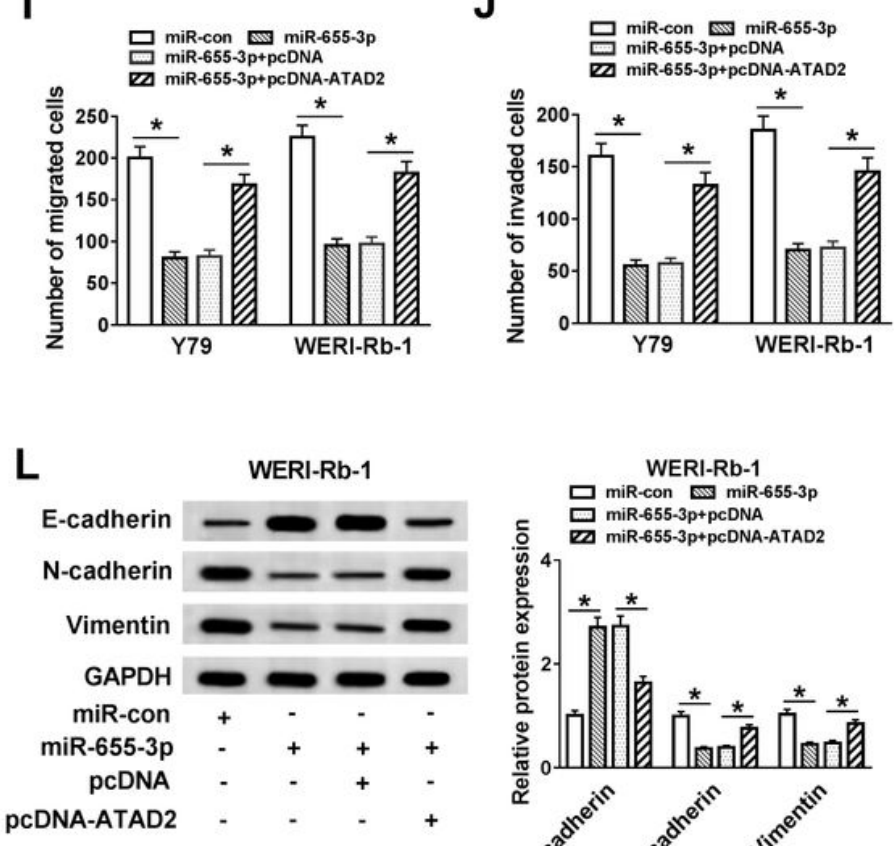

Figure 7

MiR-655-3p negatively regulated ATAD2 expression to impede cell proliferation and impel apoptosis in Y79 and WERI-Rb-1 cells. (A-L)The Y79 and WERI-Rb-1 cells were transfected with miR-con, miR-655-3p, miR-655-3p + pcDNA or miR-655-3p + pcDNA-ATAD2. (A-C) The levels of ATAD2 mRNA and protein were detected via qRT-PCR and Western blot assay. (D-E)The cell viability was detected by CCK8 assay. (F) The apoptotic rate was measured through flow cytometry. (G-H and K-L) The protein levels of p21, CyclinD1, Bcl-2, cleaved-casp-3, E-cadherin, N-cadherin, and Vimentin were assessed via Western blot assay. (I-J) The migrated and invaded abilities were estimated by Transwell assay. ${ }^{*} \mathrm{P}<0.05$. 
A

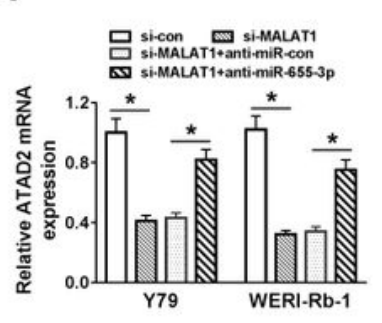

E

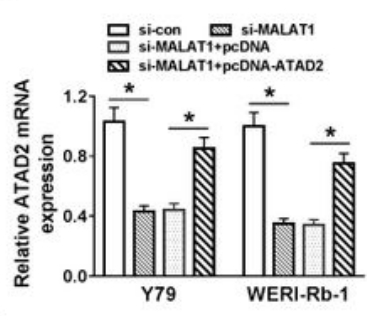

I

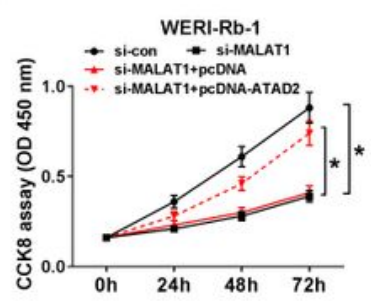

L

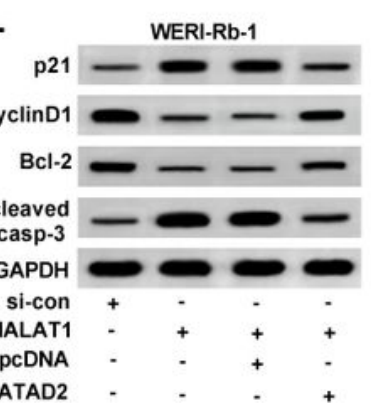

0

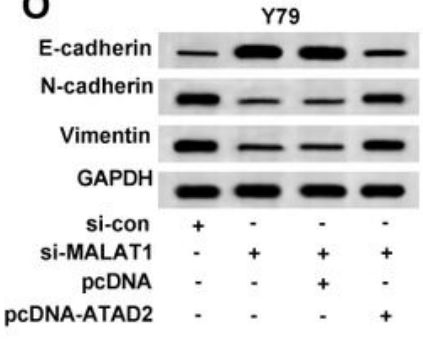

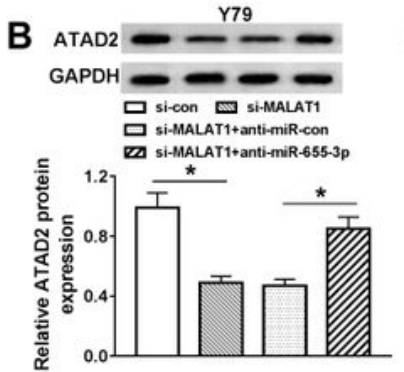

F
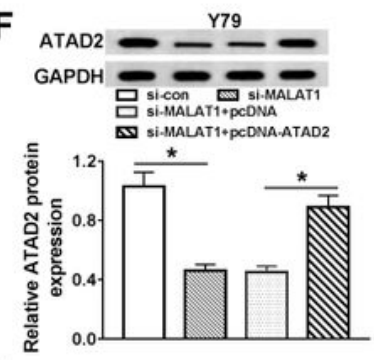

J
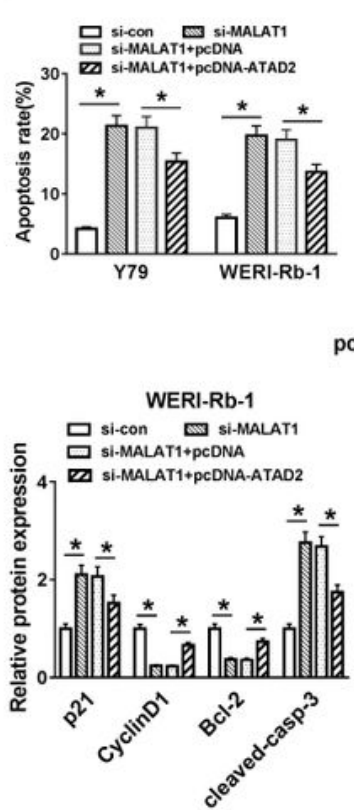

Y79

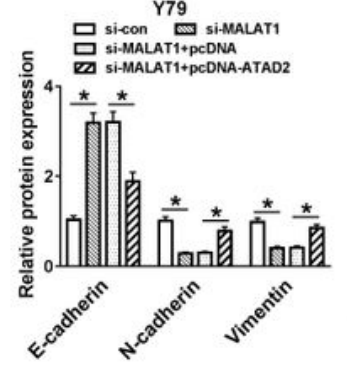

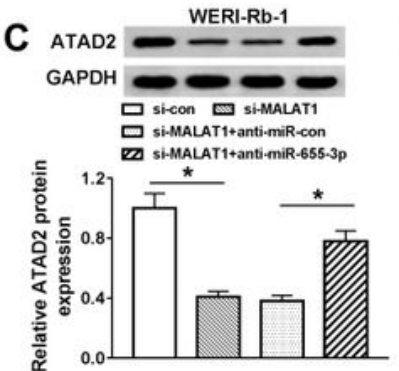

D
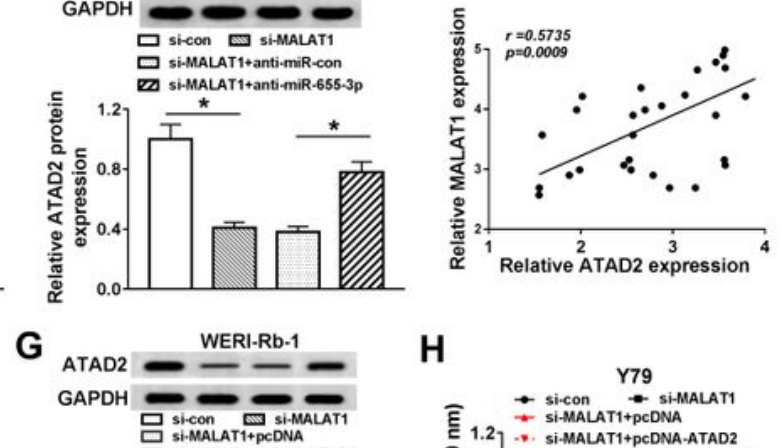

H

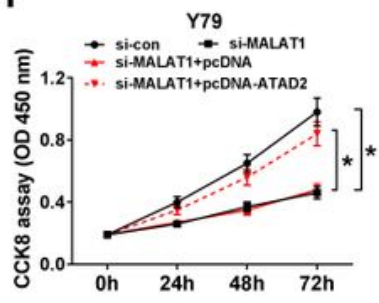

Y79

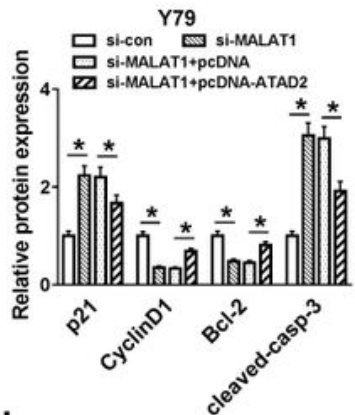

N

M

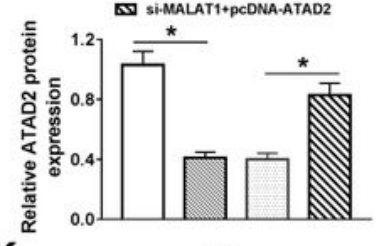

K
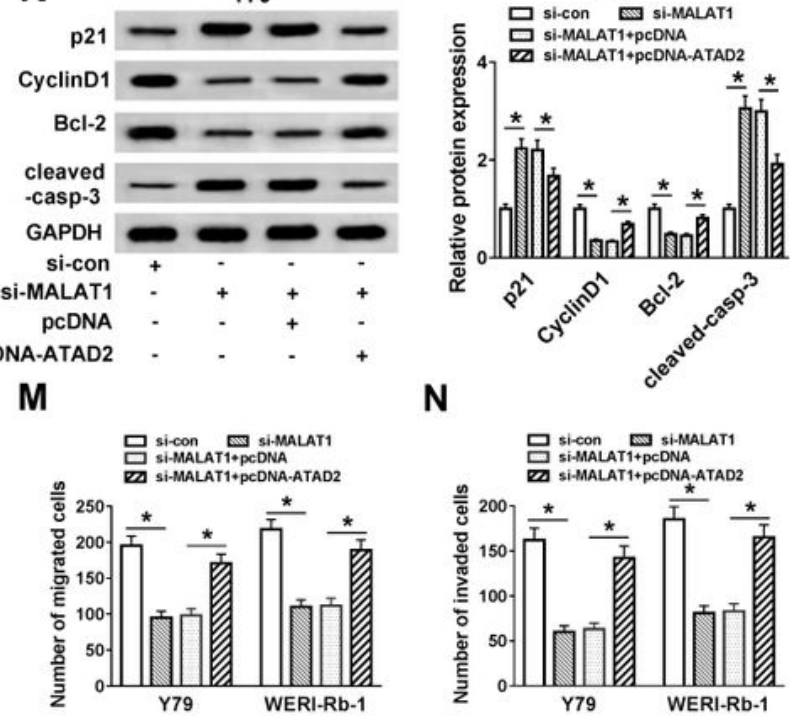

\section{Figure 8}

MALAT1 positively regulated ATAD2 expression to affect cell behaviors in RB by sponging miR-655-3p. (A-C) The mRNA and protein levels of ATAD2 in Y79 and WERI-Rb-1 cells transfected with si-con, siMALAT1, si-MALAT1 + anti-miR-con, or si-MALAT1 + anti-miR-655-3p was measured via qRT-PCR and Western blot assay. (D) The correlation between MALAT and ATAD2 was validated by Pearson correlation analysis. (E-P) The Y79 and WERI-Rb-1 cells were transfected with si-con, si-MALAT1, si-MALAT1 + 
pcDNA, or si-MALAT1 + pcDNA-ATAD2. (E-G) The levels of ATAD2 mRNA and protein was tested by qRTPCR and Western blot assay. $(\mathrm{H}-\mathrm{I})$ The cell viability was measured by CCK8 assay. $(\mathrm{J})$ The apoptotic rate was assessed using flow cytometry. (K-L and O-P) The protein levels of p21, CyclinD1, Bcl-2, cleavedcasp-3, E-cadherin, N-cadherin, and Vimentin were tested via Western blot assay. (M-N) The migrated and invaded abilities were estimated by Transwell assay. ${ }^{*} P<0.05$.
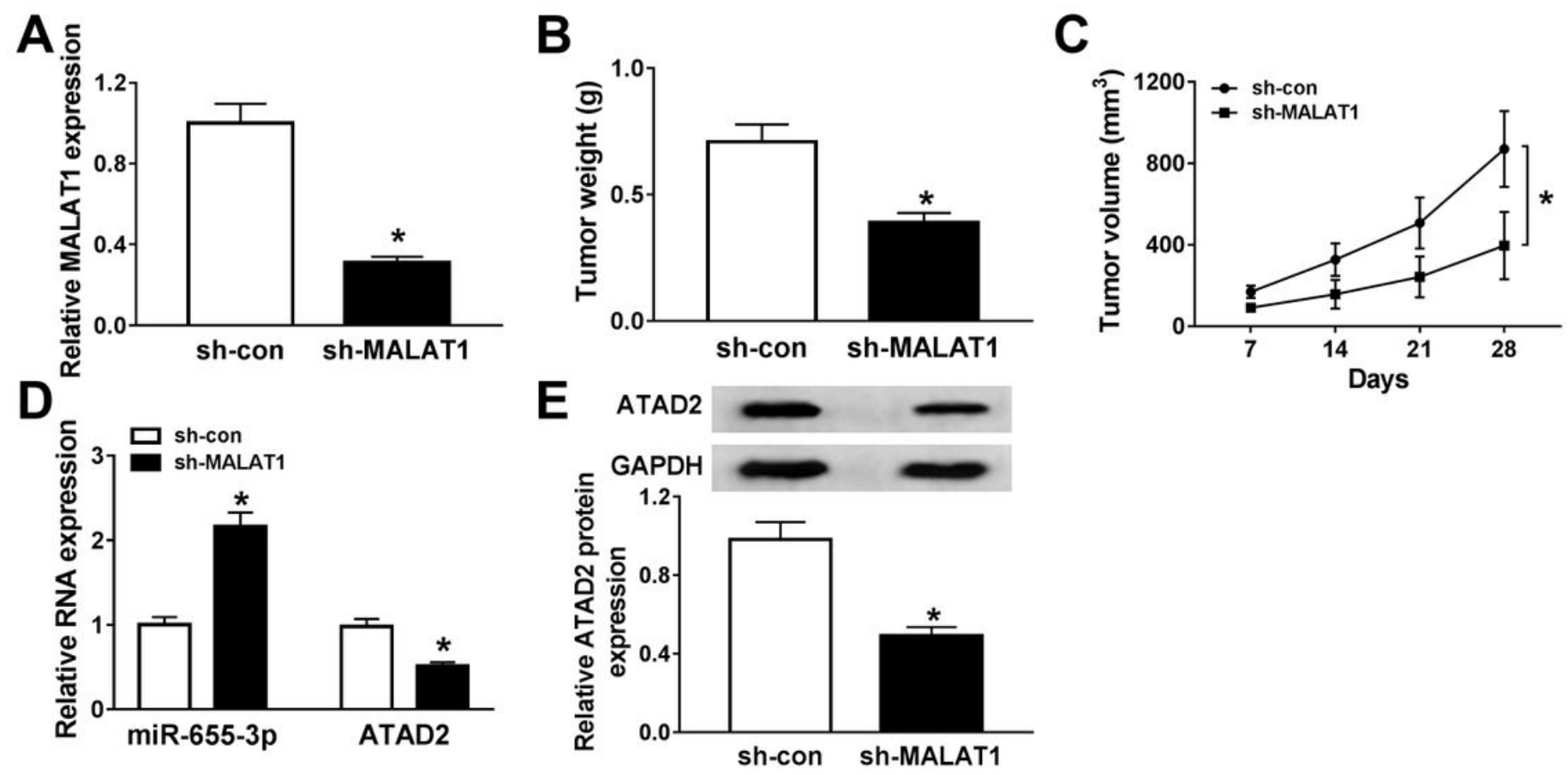

\section{Figure 9}

MALAT1 depletion impeded xenograft tumor growth in vivo. (A) The level of MALAT1 in Y79 cells transfected with sh-MALAT1 or sh-con was detected by qRT-PCR. (B-E) The nude mice were injected with Y79 cells stably transfected with sh-MALAT1 or sh-con. (B-C) The weight and volume of xenograft tumor were displayed. (D) The levels of miR-655-3p and ATAD2 were detected via qRT-PCR. (E) The protein level of ATAD2 was tested via Western blot assay. ${ }^{*}<<0.05$. 